\title{
Risk factors of post operative pyrexia in infants and children at Assuit University Children Hospital.
}

\author{
Sabah Abd El-Aziz Ahmed Shafea, Almoutaz Ahmed Eltayeb, Hekmat Ibrahim Abd EL-Kreem \& Marzoka \\ Abd El-Aziz Gadallah
}

Master Degree in Pediatric and Premature Nursing Assuit University, Children Hospital.

Assistant Professor of Pediatric Surgery Faculty of Medicine, Assuit University.

Assistant professor of pediatric nursing Faculty of nursing, Assuit University.

Assistant Professor of Pediatric Surgery Faculty of Nursing, Assuit University.

\begin{abstract}
Post operative pyrexia is one of the most common problems seen in the post operative ward. Aims: to identify risk factors of post operative pyrexia in infants and children at Assuit University Children Hospital. Subjects and Method: Descriptive research design was used. The present study was conducted in the pediatric surgical unit at Assuit University Children Hospital. The sample included two groups. Group I pyrexia group 212 children with pyrexia, group II non pyrexia group 76 of children who have no pyrexia. One tool was used to collect the required data Results: blood transfusion was given to approximately half of group I compared to only (6.6\%) of group II. More than two fifths of children had signs of wound infection, urinary catheterizations respectively in group I compared to $13.2 \%$ children in group II respectively. The majority of children received intravenous fluid after operation in group I compared to (14.6\%) of children group II. Conclusion: Risk factors of post operative pyrexia are wound infection, respiratory problem, urinary tract infection, blood transfusion, drains and catheters, medications and intravenous line infection. Recommendations: Increasing mother's and nurses awareness related to risk factors of post operative pyrexia, health education about importance of hand washing before and after wound dressing and giving medication.
\end{abstract}

\section{Key words: prevalence, risk factors \& pyrexia.}

\section{Introduction}

Post operative pyrexia is one of the most common problems seen in the post operative ward. In general can be define as.The incidence of post operative pyrexia is remarkably high, but the reported numbers vary with type and duration of surgery, patient age, pre- existing inflammation and surgical site. However, published incidence rates ranged widely from $14 \%$ to $91 \%$ depending on how pyrexia was defined and the pediatric children population of the study (Litman, 2006).

Post operative pyrexia is a common condition challenging doctors to find the right diagnosis, because it can be a hallmark of serious underlying conditions. Between $40 \%-50 \%$ of pediatric patients develop pyrexia after surgery depending on type of surgery but only a small percentage turn out to be due to infection and can also be brought on by the inflammation involved in the surgery itself and does not always require medical attention (Pile 2006).

The differential diagnosis of post operative pyrexia includes infectious and noninfectious conditions that occur following surgery. Pyrexia may arise due to a surgical site infection (SSI), or from other hospitalrelated conditions, including nosocomial pneumonia, urinary tract infection, drug pyrexia, and deep vein thrombosis. In evaluating a post operative patient with pyrexia, it is important to consider a broad differential diagnosis, and not to assume that pyrexia is due to infection (Geerts, et al., 2008 ).

Post operative pyrexia is probably due to intraoperative tissue trauma with subsequent release of endogenous pyrogen into the blood stream has made clinically significant observation. Seventy two percent of pyrexia occurring within the 48 hours of surgery are non-infection mediated, while wound, urinary tract, and respiratory infections occur later than 48 hours. Chronic fever is a normal fever that lasts unusually long and is untypical for the cause. if temperature is constantly at $100^{\circ} \mathrm{F}$ over a long period of time, you may suffer from chronic fever. (Chang et al., 2006).

Third-day surgical pyrexia has been used to describe pyrexia occurring on the third post operative day as a result of an infection at the intravenous site. Such pyrexia are not limited to the third day and may result from contaminated intravenous fluid as well as infection at the catheter site. Inflammation may be absent from the intravenous site, making the diagnosis more difficult. Therapy consists of removing the catheter, culturing its tip, and obtaining several blood cultures. (Blum, et al., 2013 ). 
Pyrexia above $38^{\circ} \mathrm{C}$ is common in the first few days after major surgery. Most early post operative pyrexia is caused by the inflammatory stimulus of surgery and resolves spontaneous. However, post operative pyrexia can be a manifestation of a serious complication as deep vein thrombosis (blood clots) and post operative pneumonia (Thomas \& Clyburn, 2010).

The surgeon must individualize care of each wound, but the sterile dressing placed in the operating room is generally left intact for 24 hours unless signs of infection (as increasing pain, erythema, or discharge) develop. After 24 hours. The site should be checked twice a day if possible, for signs of infection and if it occurs, the wound is explored and drained. Antibiotics, whither systemic, topical or both may be required. Topical antibiotics are usually not helpful. Tube drains if present must be monitored for quantity and quality of the fluid collected. Skin sutures, staples and other closures are usually left in place for 7 days or longer depending on the site (Robert \& Johuson, 2009).

The pediatric nurse plays an important role for surgical infants and children intra operatively and post operatively. Once the infants or children are is transferred to the operating unit they become the nurse's responsibility. They have to monitor patient's condition closely. They to report anything that are out of the ordinary to the physician or anesthesiologist. The nurses monitor children closely for signs of shock, hemorrhage, inadequate oxygenation and hyperthermia. Blood pressure, heart rate, oxygen saturation and breathing patterns will also be closely monitored. In addition, they started carefully document each vital sign, assess the extent of pain and administer pain relieving medication according to the doctor's or hospital's order. Another role of the post operative nurse is to carefully and accurately note any and all medications given to the children during the post operative period and be alert to any side effect related to those medications (Hockenberry et al., 2007).

\section{Significant of the problem}

Post operative pyrexia is a common condition challenging doctors to find the right diagnosis, because it can be a hallmark of serious underlying conditions. Between $40 \%-50 \%$ of pediatric patients develop pyrexia after surgery depending on type of surgery but only a small percentage turn out to be due to infection and can also be brought on by the inflammation involved in the surgery itself and does not always require medical attention (Pile., 2006). The incidence of post operative pyrexia for infant and children in Assuit calculated from the files of Pediatric Surgical Unit Assuit University Children Hospital was as high as $73.6 \%$ in Year 2012.

\section{Aim of the Study}

This study aims to identify risk factors of post operative pyrexia in infants and children at Assuit University Children Hospital.

What are the risk factors of post operative pyrexia among infants and children

\section{Subjects and Method}

Research Design

A descriptive research design was used to meet the aim of the study.

Setting

The study was conducted in Surgical Unit at Assiut University Children Hospital.

\section{Subjects}

It consists of mother having infants and children with or without post operative pyrexia attending the previously mentioned during a period of six months from May 2012 to October 2012. A total of (288) infants and children were divided into two groups: 212 infants and children with pyrexia within 24hours and 76 of them without pyrexia. It measure 4 times per days and related children condition of post operative

\section{Tool of the Study}

\section{Risk factors of post operative pyrexia tool}

Structured interview questionnaire sheet for infants and children. It was developed by the researcher. It consists of three parts

First Part: Includes after through review the literature characteristics of infants and children as ; name, age, sex, residence and birth order.

Second Part: Clinical data of infants and children such as date of admission, operation and discharge, time of the hospital stay, clinical diagnosis and temperature report.

Third Part: Risk factors of post operative pyrexia. It included:- type of surgery, type of anesthesia, post operative medications, postoperative blood transfusion, duration of catheter insertion, surgical drains, post operative complications, signs of wound infections, signs of respiratory distress, and urinary symptoms.

\section{Method of Data Collection}

1-An official permission will be obtained from the chairmen of pediatric Surgical Unit at Assiut University Children Hospital.

2- Written consent was obtained from mothers or older children. The work was carried out by the investigator herself.

3- Validity of the tool was done after become receive by five experts in the pediatric nursing field

4- Reliability of the tool was estimated by Alfa cronbach's test. 
5- The pilot Study: Was carried out on $10 \%$ of mother having with or without children post operative pyrexia to test the clarity and applicability of the tool and estimate the length of time required to fill in the standard interview tool. According to the results of the pilot study, the essential modifications will done and the final form was developed, were excluded from the sample.

6- Field of the Work: a) The period of data collection started from May to October 2012. b) Every mother was interviewed individualler after explanation of the aim of the study aim. c) Mothers were reassured that information obtained is confidential and used only for the purpose of the study. d) The time used for filling each sheet ranged between 20-30 minutes, and daily record temperature was continuous until discharge or death.

7- Data collection was the responsibility of the researcher.
8- Assessments of risk factors for infants and children in pyrexia group were done by the researcher from first day post operative time to the discharge using tool 1.

9- Temperature recorded from the initial post operative pyrexia until discharge.

10- Place of follow up included only the pediatric surgical unit.

\section{Statistical Analysis of Data}

Data entry was done using Epi-Info 6.04 computer software package, while statistical analysis was done using SPSS Version 12.0 statistical software package. Data was presented using descriptive statistics in the form of frequencies and percentages for qualitative variables, and means and standard deviations using independent $\mathrm{T}$ test for quantitative variables. Qualitative variables were compared using chi-square test. statistical significance was considered at p-value $<0.05$.

\section{Results}

Table (1): Personal characteristics of studied infants and children.

\begin{tabular}{|c|c|c|c|c|c|}
\hline Items & \multicolumn{2}{|c|}{$\begin{array}{c}\text { Pyrexia group } \\
(\text { group I) } n=212\end{array}$} & \multicolumn{2}{|c|}{$\begin{array}{l}\text { Non pyrexia group } \\
\text { (group II) } \quad n=76\end{array}$} & \multirow[t]{2}{*}{ P. value } \\
\hline 1-Age & No. & $\%$ & No. & $\%$ & \\
\hline$<1$ years & 75 & 35.3 & 16 & 21.1 & \multirow{4}{*}{$0.022 *$} \\
\hline $1-$ & 58 & 27.4 & 36 & 47.4 & \\
\hline $6-$ & 46 & 21.7 & 15 & 19.7 & \\
\hline $12-15$ years & 33 & 15.6 & 9 & 11.8 & \\
\hline Mean \pm SD & \multicolumn{2}{|c|}{$2.63 \pm 1.52$} & \multicolumn{2}{|c|}{$4.7 \pm 4.5$} & $0.001^{*}$ \\
\hline $2-\operatorname{Sex}$ & No. & $\%$ & No. & $\%$ & \\
\hline Male & 146 & 68.9 & 61 & 80.3 & \multirow{2}{*}{0.058} \\
\hline Female & 66 & 31.1 & 15 & 19.7 & \\
\hline
\end{tabular}

*statistical significant differences

Table (2) : Relationship between pyrexia and non pyrexia groups of infants and children according to the indications of surgery, type of surgery \& wounds.

\begin{tabular}{|c|c|c|c|c|c|}
\hline \multirow{2}{*}{ Items } & \multicolumn{2}{|c|}{ Pyrexia group $(n=212)$} & \multicolumn{2}{|c|}{ Non-pyrexia $(n=76)$} & \multirow{2}{*}{ P. value } \\
\hline & No. & $\%$ & No. & $\%$ & \\
\hline \multicolumn{6}{|l|}{ 1- Indication of surgery } \\
\hline Elective surgery & 97 & 45.8 & 62 & 81.6 & \multirow{2}{*}{$0.001 *$} \\
\hline Emergency surgery & 115 & 54.2 & 14 & 18.4 & \\
\hline \multicolumn{6}{|l|}{ 2-Type of surgery } \\
\hline Major operation & 116 & 54.7 & 5 & 6.6 & \multirow{3}{*}{$0.001 *$} \\
\hline moderate operation & 92 & 43.4 & 71 & 93.4 & \\
\hline Minor operation & 4 & 1.9 & 0 & 0.0 & \\
\hline \multicolumn{6}{|l|}{ 3- Type of wounds } \\
\hline Clean wounds & 128 & 60.4 & 75 & 98.7 & \multirow{4}{*}{$0.001 *$} \\
\hline Clean contaminated wounds & 38 & 17.9 & 0 & 0 & \\
\hline Contaminated wounds & 23 & 10.8 & 0 & 0.0 & \\
\hline Dirty wounds & 23 & 10.8 & 0 & 0.0 & \\
\hline
\end{tabular}

*statistical significant differences 
Table (3): Distribution of pyrexia and non pyrexia according to presence of wound infection and surgical drains.

\begin{tabular}{|c|c|c|c|c|c|}
\hline \multirow{2}{*}{ Items } & \multicolumn{2}{|c|}{ Pyrexia group $(n=212)$} & \multicolumn{2}{|c|}{ Non-pyrexia $(n=76)$} & \multirow{2}{*}{ P. value } \\
\hline & No. & $\%$ & No. & $\%$ & \\
\hline Presence of Signs of wound infection & 84 & 39.6 & 0 & 0.0 & $0.001 *$ \\
\hline \multicolumn{6}{|l|}{ Surgical drains } \\
\hline Suction & 41 & 19.4 & 0 & 0.0 & $0.001 *$ \\
\hline Closed & 123 & 58.0 & 8 & 10.5 & $0.001^{*}$ \\
\hline No drains & 48 & 22.6 & 68 & 89.4 & $0.001 *$ \\
\hline
\end{tabular}

*statistical significant differences

Table (4) : Distribution of pyrexia and non pyrexia infants and children according to exposure and duration of urinary catheterizations.

\begin{tabular}{|c|c|c|c|c|c|}
\hline \multirow{2}{*}{ Items } & \multicolumn{2}{|c|}{ Pyrexia group $(n=212)$} & \multicolumn{2}{|c|}{ Non-pyrexia $(n=76)$} & \multirow{2}{*}{ P. value } \\
\hline & No. & $\%$ & No. & $\%$ & \\
\hline \multicolumn{6}{|l|}{ 1- Exposed to Urinary catheterizations } \\
\hline Infants and children with urinarycatheterizations & 121 & 42.9 & 10 & 13.2 & $0.001 *$ \\
\hline 2- Duration of catheterization & No. & $\%$ & No. & $\%$ & P. value \\
\hline 1- & 15 & 12.4 & 2 & 20.0 & 0.421 \\
\hline $3-$ & 42 & 34.7 & 1 & 10.0 & 0.105 \\
\hline $5-$ & 40 & 33.1 & 3 & 30.0 & 0.439 \\
\hline 7 days and more & 24 & 19.8 & 4 & 40.0 & 0.137 \\
\hline Total & 121 & 42.9 & 10 & 13.2 & $0.001^{*}$ \\
\hline
\end{tabular}

*statistical significant differences

Fig (1) Exposure of pyrexia and non pyrexia infants and children to blood transfusion.

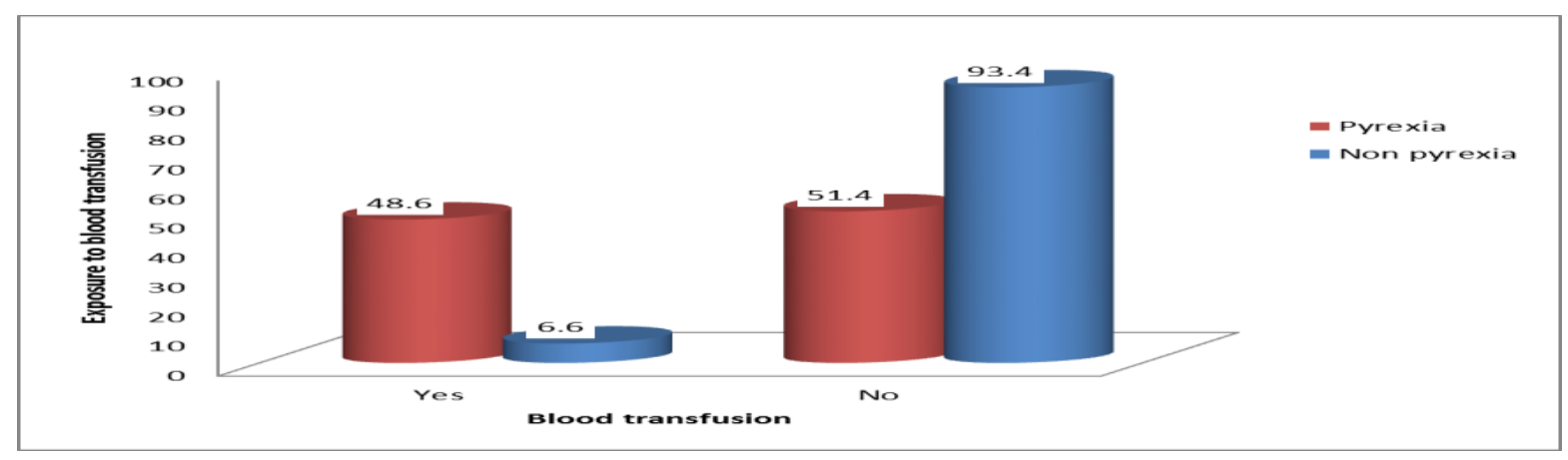

Fig (2) Hospital stay among pyrexia and non pyrexia infants and children .

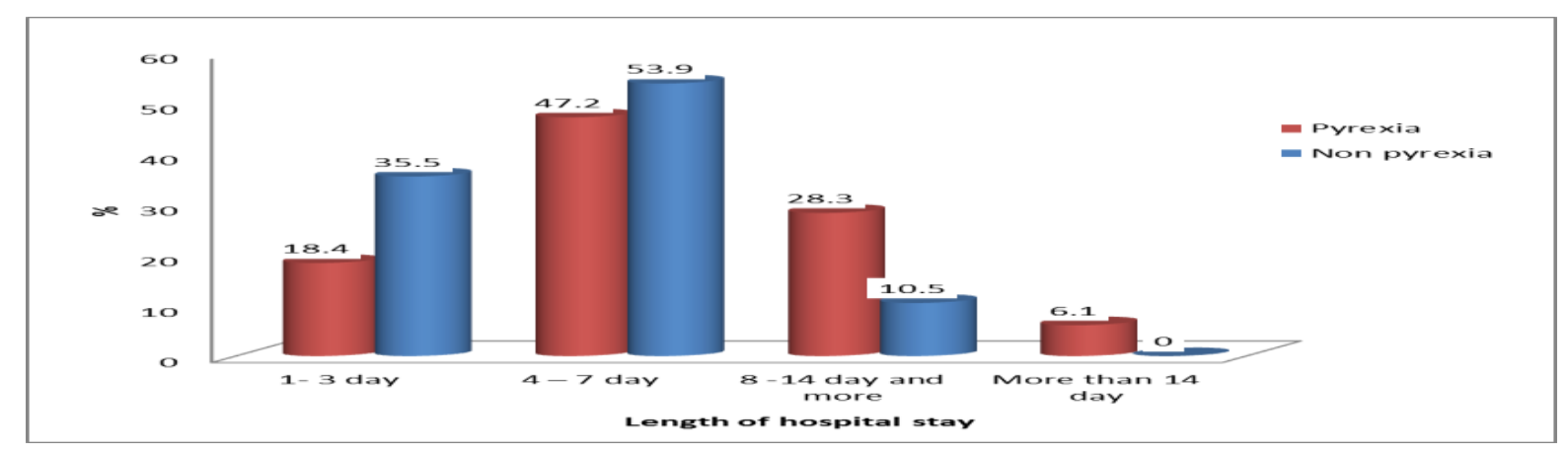


Fig (3) Relationship between both groups regarding to Parenteral nutrition

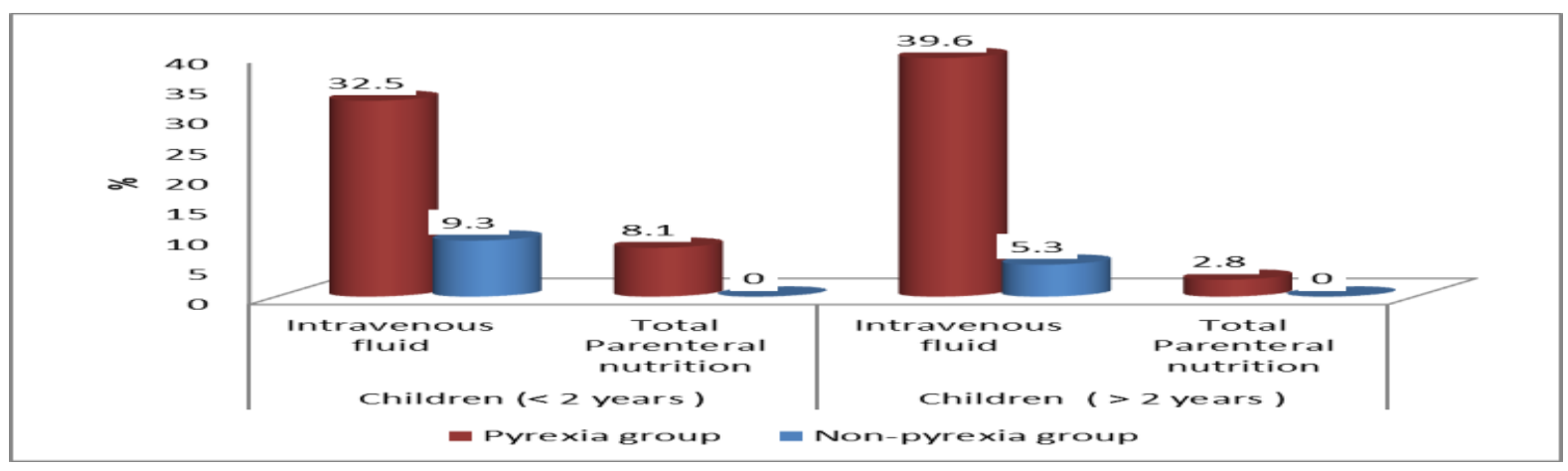

Fig (4) Distribution of pyrexia group related to period of fever.

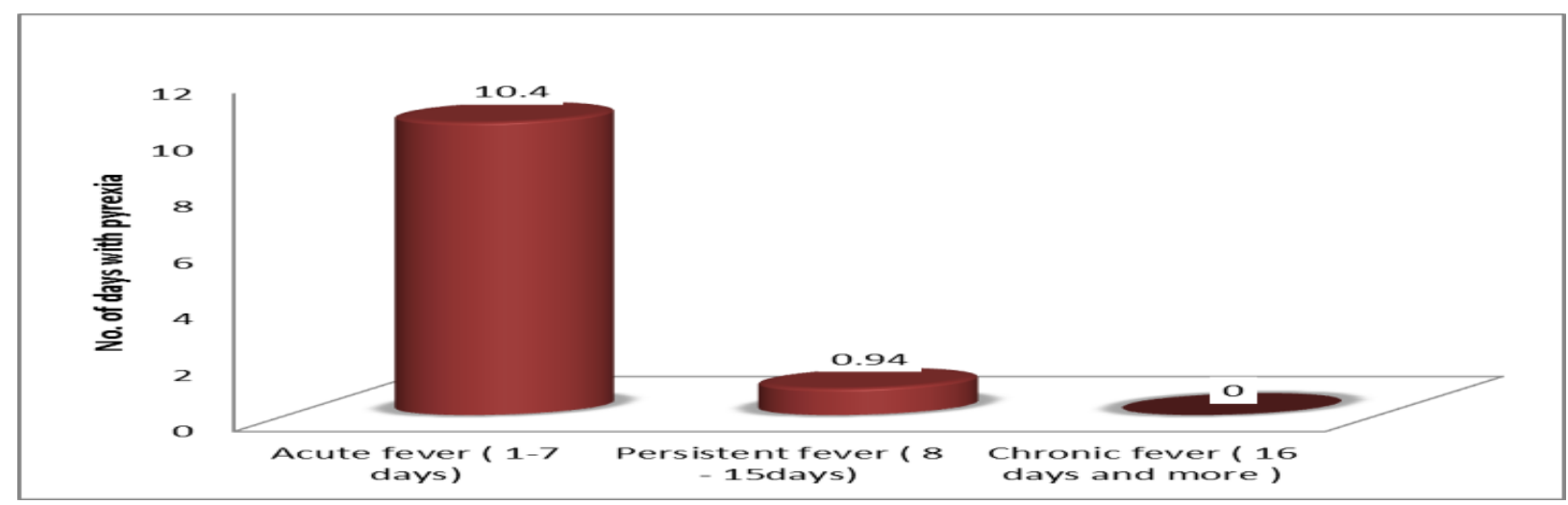

Table (1) : showed Personal characteristics of studied infants and children. Statistically significant differences were found between children in the two groups regarding their age and sex. More than one third $(35.4 \%)$ of infants in the pyrexia group aged < one year compared to $(21.1 \%)$ of them in the non pyrexia group while more than one forth $(27.4 \%)$ of children in the pyrexia group aged from 1 - 6 years compared to nearly half $(47.4 \%)$ of them in the non pyrexia group. Mean age of studied children is 2.63 \pm 1.52 years in the pyrexia group and $4.7 \pm 4.5$ years in the non pyrexia group. As regards the sex of studied children the majority of them in the two groups were males $(68.9 \%)$ in the pyrexia group and $(80.3 \%)$ in the non pyrexia group.

Table (2) : Illustrates relationship between both groups of infants and children according to the indication of surgery, type of surgery and wound, As regard indication of surgery it was more than half $(54.2 \%)$ of infants and children undergone emergency surgery in the pyrexia group compared to $(18.4 \%)$ of infants and children in the non pyrexia group. According to type of surgery more than half $(54.7 \%)$ of infants and children undergone major operation in the pyrexia group compared to only $(6.6$ $\%)$ in the non pyrexia group. However, only $(1.9 \%)$ of children undergone minor operation in the pyrexia group compared to zero \% in the non pyrexia group. More than half of children (60.4\%) with clean wounds developed pyrexia compared to majority of them $(98.7 \%)$ with no pyrexia a small percent of children with contaminated and dirty wounds $(10.8 \%)$ had pyrexia in the pyrexia group compared to no one child in the non pyrexia group.

Table (3) : Relationship between both groups regarding signs of wound infection and surgical drains. As regards signs of wound infection approximately two fifths (39.6\%) of children in the pyrexia group had signs of wound infection compared to no one child in the non pyrexia group. As regards surgical drains, closed surgical drains represented more than one half $(58.0 \%)$ of children in the pyrexia group compared to $(10.5 \%)$ of them in the non pyrexia group, while no drains represented $(22.6 \%)$ of children in pyrexia group compared to $(89.4 \%)$ of children in non pyrexia group.

Table (4) : Relationship of fever according to urinary catheterizations. Statistically significant difference was found between the two groups regarding urinary catheterizations; $\mathrm{P}=0.001$ More than two fifths $(42.9 \%)$ of infants and children in the pyrexia group had urinary catheterizations compared to (13.2\%) of them in the non pyrexia group. According to duration 
of catheterization it was observed that there is no statistically significant difference was found between children in the two groups.

Fig (1) : Demonstrates percentages of blood transfusion among infants and children in both groups. Highly statistically significant difference was found between the two groups, approximately half $(48.6 \%)$ of infants in pyrexia group received blood transfusion compared to only $(6.6 \%)$ of them in the non pyrexia group.

Fig (2) : Shows duration of hospital stay among infants and children in the pyrexia and the nonpyrexia groups. Statistically significant differences were found between two groups as regards the duration of hospital stay with significantly higher frequencies of staying either $4-7$ day or $8-14$ days in the pyrexia group than in the non pyrexia group $(47.2 \%$ vs. $28.3 \%$ and $53.9 \%$ vs. $10.5 \%$, respectively. (P values $<0.001$ for both). While only ( $6.1 \%$ ) of children stayed more than 14 days in the pyrexia group compared to no single child in the non pyrexia group.

Fig (3) : Illustrates Relationship between the pyrexia and the non pyrexia groups of children regarding parenteral nutrition during the first three days after operation, it was observed that Intravenous fluid represented nearly one third $(32.5 \%)$ of children less than 2 years in the pyrexia group compared to only (9.3\%) among children in the non pyrexia group, while total parenteral nutrition represented $(8.1 \%)$ among children in the pyrexia group compared to no one child in the non pyrexia group. (P-value 0.01 and 0.05 respectively). In children above two years, Intravenous fluid represented more than one third $(39.6 \%)$ of infants and children in the pyrexia group compared to $(5.3 \%)$ of them in the non pyrexia group. (P-value 0.001).

Fig (4) : Demonstrates period of fever among infants and children in the pyrexia group, it was found that $(10.4 \%)$ of infants and children had Acute fever followed $(0.94 \%)$ of them had persistent fever $(0.94 \%)$ and no one had chronic fever .

\section{Discussion}

Based on results sited above, it was observed that the presence of pyrexia is decreased with increasing age of the child. More than one third of infants and children aged less than one years and more than one fourth of infants and children aged 1- 6 years had pyrexia. This may be explained by the infants liability to infection due to low immunity compared to children aged more than one year as shown in table (1). These data disagreed with (Rudra et al., 2006) who found that pyrexia increase with increasing age.
According to the gender, the present study found that more than two thirds of infants and children were males in the pyrexia group compared to more than three quadrant $(80.3 \%)$ in non pyrexia group as shown in table (1). The results of the present study agreed with (Awadh et al., 2007) who stated that gender was not a risk factor to post operative pyrexia and however, agreed with (Ahmed et al., 2009) who found that $62.4 \%$ were males.

The present study revealed that major operation represented more than half of febrile infants and children in the pyrexia group compared to low percent of infants and children in the non pyrexia group as shown in table (2). These results are in agreement with those obtained by (Pile, 2006 \& Blum et al., 2007) who found that major surgical procedures carry definite risks for the pediatric children such as infection at the site of the surgery, hemorrhage, or complications from the general anesthesia used.

Regarding type of wound it was observed that more than ten percent $(10.8 \%)$ of infants and children had dirty wounds in the pyrexia group compared to those in the non pyrexia group as shown in Table (2), these results are congruent with those obtained by Vermeulen et al., 2007) who found that presence of post-operative pyrexia is highest in infants and children with dirty wound

The present study revealed that more than one third of infants and children with the pyrexia group had signs of wound infection compared zero \% of infants and children with the non pyrexia group as shown in Table (3) : This result agreed with (Sikora, 2003) who found that signs of wound infections in and around the incision is associated with pyrexia and agree with (Paul \& Marik, 2008) who found that wound infection is a cause of pyrexia and also agreed with (Geerts et al., 2008) who found that the post operative day on which a given wound infection becomes apparent and the local signs of sepsis produced by the infection vary according to the organism and the concomitant use of antibiotics..

Regarding the surgical drainage it was observed that the closed surgical drainage represented more than one half $(58.0 \%)$ of infants and children in the pyrexia group compared to only $(10.5 \%)$ of those in the non pyrexia group as shown in table (3), This result is in agreement with (Garibaldi et al., 2000) who found that drains can act as a point of access for infection

Regarding urinary catheterization of post operative pyrexia among infants and children it was observed that more than two fifths $(42.9 \%)$ of infants and children used urinary catheterization in the pyrexia group compared to $(13.2 \%)$ of them with the non pyrexia group. Moreover the duration of 
catheterizations of infants and children in the pyrexia and the non pyrexia groups was $(33.1 \%)$ and $(19.8 \%)$ respectively, from (5-7) days and more than 7 days respectively as shown in table (4). These results are is in congruent to (Paul \& Marik, 2008) findings who found that the duration of catheterization is the most important risk factor for the development of nosocomial cystitis or pyelonephritis and results obtained by (Lenzt, 2012) who found that Patients developed pyrexia as a result of an indwelling catheter, such as plastic intravenous (IV) lines or foley catheters. Moreover, (Sikora, 2003) found that urinary tract infection (UTI) was likely from the urinary catheter inserted during surgery. Invasive procedure increase higher risk of infection. non invasive procedure is recommended $40-50 \%$ infection due to hand washing.

Blood transfusion is one of risk factors of post operative pyrexia in infants and children, the present study observed that nearly half of children $(48.6 \%)$ received blood transfusion in the pyrexia group compared to only $(6.6 \%)$ of them in the non pyrexia group as shown in figure (1). This results are agreed with findings of Paul \& Marik, (2008) who found that pyrexia associated with transfusion of blood products, particularly red blood cells and platelets, occurs most frequently in pediatric patients who have received multiple transfusions. Although blood transfusions can be life-saving, they are not without risks. Infections were once the main risk, but they have become extremely rare with careful testing and donor screening. Transfusion reactions and other non-infectious problems are now more common.

The present study observed that $(28.3 \%)$ of infants and children in the pyrexia group had duration of hospital stay ranged from 8 -14 days and compared to $(10.5 \%)$ of them in the non pyrexia group as shown in figure (2). This result is agreed with result of (Lentz, 2012) who found that pyrexia increase the duration of the post operative hospital stay. Infection occurs up to 48 hours after hospital admission

According to parenteral nutrition during the first three days after operation the present study observed that the majority of infants and children $(83 \%)$ in the pyrexia group received total parenteral nutrition compared to $(14.6 \%)$ of infants and children in the non pyrexia group as shown in figure (3) (Ahmed et al., 2009) found that $71.5 \%$ of infants and children with post operative pyrexia received total parentral nutrition. in addition (Christensen \& Kockrow, 2006) stated that third-day surgical pyrexia has been used to describe pyrexia occurring as a result of an infection at the intravenous site. Such pyrexia are not limited to the third day and may result from contaminated intravenous.
Regarding the period of fever the present study was observed that $10.4 \%$ of infants and children with the pyrexia group had acute pyrexia (between one to three days after the operation) as shown in figure (4). This result is agreed with (Hendershot \& Colapinto, 2006) results who stated that the highest incidence of post operative pyrexia is between one and three days after the operation.

\section{Conclusion}

The most common risk factors of post operative pyrexia in the study were. wound infection, type and duration of surgery, blood transfusions, intravenous line infections, drains and urinary catheters.

\section{Recommendations}

\section{The Study Recommended That}

The use of aseptic techniques during insertion of intravenous, cannula, obtaining blood sample, medication, urinary catheter, blood transfusion or vein puncture, nutrition, wound infection and drainage, to minimize transport of infection.

The hospital should keep written policies and procedures about infection control precautions especially hand washing, use of gloves, gown, mask and management of sharp items in each hospital ward and reviewed every two years.

Infection control committee should provide physicians, nurses and auxiliary persons with pre service and ongoing in-service training programs regarding universal infection control precautions.

Health education and increase awareness to the nurses about the risk factors of post operative pyrexia and procedures related to care of children with wound infection and post operative pyrexia

Record intake and output chart in post operative febrile children to prevent dehydration, check for drainage and catheter for proper functioning and remove drainage and catheter if possible to decrease source of infection.

\section{References}

1. Litman R., Flood C., Tobin J., \& Kaplan R., (2006): Post operative Malignant Hyperthermia. Relative Incidence and Clinical Characteristics. Anesthesiology, 18,Pp.105:115.

2. Pile J., (2006): Evaluating Post operative Fever. A focused Approach. Cleveland Clin Medicine Journal. 73( 1):62-6.

3. Geerts W., Pineo G., \& Heit J., (2008): Prevention of Venous Thromboembolism, American College of Chest Physicians Evidencebased Clinical Practice Guidelines, Chest J, 133(6):381-453. 
4. Chang A., Henderson E., \& Colapinto N., (2006): Minimizing Complications Related to Fever in the Post Operative Pediatric Surg Patient. Pediatr surg Nurs J, 23:75.

5. Blum J., Stentz M., \& Dechert R., (2013): Preoperative and Intraoperative Predictors of Postoperative Acute Respiratory Distress Syndrome in a General Surgical Population. Anesthesiology;118(10):19-29.

6. Thomas D., Wee M., \& Clyburn P., (2010): Blood transfusion and the anaesthetist: management of massive haemorrhage. Anaesthesia;65(11) :1153-61.

7. Robert G., \& Johuson M., (2009): Topic of Care of Surgical patients. Post Operative Care. $2^{\text {nd }}$ edition, Pp. 2- 47.

8. Hockenberry M., Wilson D., \& Winkelstein, (2007): Wongs Nursing Care of Infants and Children, The child with respiratory dysfunction. $8^{\text {th }}$ ed., Elsevier Mosby, Canda, Pp. 1115-1117.

9. Rudra A., Pal S., \& Acharjee A., (2006): Post operative Fever. Crit Care Med Indian J,10:26471.

10. Awadh H., Hassanein F., Asker A., \& El-Din H., (2007): Early diagnosis of early neonatal sepsis in special care baby unit, Assuit University, unpublished MD thesis in pediatrics, Assuit University.

11.

12. Ahmed A., Fadel K., Mohamed S., Abd ElMegeed E., \& Mohamed E., (2009): Survillance study of nosocomial infections at neonatal intensive care unit in Pediatric Assuit University Hospital, unpublished MD thesis in pediatrics, Assuit University.

13. Vermeulen H., Storm-versloot M., Gossesens A., Speelman P., \& Legemate D., (2007): Diagnostic accuracy of routine post operative body temperature measurement. Clin Infect Dis ,40:1404- 10

14. Sikora C., (2003) : Fever in the Post operative Patient: A chilling Problem. CME Canadian journal, 93-98.

15. Paul, J., \& Marik, W., (2008) : Guideline for the Management of Pyrexia in Critically Ill Patients. Chest American College of Critical Care Medicine and the Infectious Diseases Society of America (117):855-869,

16. Garibaldi R., Burke J., Dickman M., \& Smith C., (2000) : Factors predisposing to bacteriuria during indwelling urethral catheterization. $N$ Engl Med, 211-215.

17. Christensen B., \& Kockrow E., (2006): Foundations Of Nursing. Vital signs basic nursing skills. $5^{\text {th }}$ ed, Mosby, United states of America, Pp:235-246
18. Lentz, H., (2012): Comprehensive Post Operative Counseling and Management. $6^{\text {th }} \mathrm{ed}$, Pp. 978-985.

19. Hendershot, D., \& Colapinto, E., (2006): Minimizing Complications Related to Fever in the Post Operative Pediatric Patient. Pediatr Surl NursJ;23 :75-81. 